\title{
3D placement of a new tethered UAV to UAV relay system for coverage maximization
}

\author{
Nour El-Din Safwat ${ }^{1}$, I. M. Hafez ${ }^{2}$, Fatma Newagy ${ }^{3}$ \\ ${ }^{1}$ Electronics and Electrical Communication Department, Faculty of Engineering, Ain Shams University, Cairo, Egypt \\ ${ }^{2}$ Electronics and Electrical Communication Department, Faculty of Engineering, Ain Shams University, Cairo, Egypt \\ ${ }^{3}$ Electronics and Electrical Communication Department, Faculty of Engineering, Ain Shams University, Cairo, Egypt
}

\begin{abstract}
In this paper, a new relay system that uses the UAV as a relay station between the tethered UAV and ground user (TU2U2G) is proposed. The TU2U2G system replaces the base station (BS) in the cellular system with a tethered UAV (TUAV). The TUAV is a UAV that receives power over a cable from a ground control station. It has advantages of high and variable altitude up to $(100 \mathrm{~m})$ compared to BS. In addition, it overcomes the UAV drawback of the batteries' limited capacity. After that, a 3D placement Algorithm of the UAV as a relay station in the TU2U2G system is proposed. It is presented to maximize the coverage by jointly optimizing the transmitting power and relaying distance. The TU2U2G system shows better results than the traditional cellular system in terms of optimum UAV height, maximum coverage radius, and maximum distance between BS and UAV.
\end{abstract}

Keywords: UAV network, Tethered UAVs, Placement Algorithms.

\section{Introduction}

Unmanned Aerial Vehicles (UAVs) are considered an important component of 5G. Due to the rapid deployment, mobility, and flexibility of UAVs [1], [2], they are utilized to assist the cellular system as a relay station to enhance the coverage. Several pieces of research were presented on using UAVs as aerial base stations to assist the cellular system. In [3-7], the placement of UAVs for coverage maximization is proposed. An algorithm that jointly optimizes the 3D UAV placement and path loss compensation factor to maximize the user coverage in the uplink transmission is proposed in [3]. An approach to minimize the 
total transmit power required to provide wireless coverage for indoor users is presented in [4]. A placement algorithm that maximizes the number of covered users with minimum transmission power is proposed in [5]. While the UAV Placement which maximizes the number of served users with different quality-ofservice requirements is proposed in [6]. In [7] an analytical approach is used to find the optimum altitude of the UAV for maximum coverage. In [8-11], UAV placement for throughput maximization is proposed. A joint trajectory and resource allocation algorithm for maximization of the system sum throughput is introduced in [8]. A joint transmit power and trajectory optimization algorithm to maximize the minimum average throughput is proposed in [9]. In [10], the minimum throughput of overall ground users is maximized in the downlink communication by optimizing the scheduling of multi-user communication and association jointly with the trajectory of UAVs and power control. Trajectory and resource allocation are jointly optimized for maximizing the system energy efficiency in [11]. An algorithm to maximize the downlink sum-rate of the network is proposed in [12]. An algorithm for UAV placement based on sparse recovery is presented in [13]. However, all these works consider only the power constraints of the communication link between UAV and the ground user mobile station (MS) and don't consider the power constraints of the communication link between UAV and the BS. Otherwise, researches that consider both links is presented in [14-22]. [14-18] are proposed for throughput maximization. 3D placement of UAV as a relay station for maximizing the average achievable rate through the one-dimensional linear search is proposed in [14]. In [15], the optimization problem is formulated to maximize the system throughput. An algorithm to find the UAV optimal position based on LOS information to maximize the end-to-end throughput is proposed in [16] . [17] explores the relationship between system throughput and placement of a UAV acting as a communication relay. An approach to jointly optimize throughput and the UAV's trajectory is presented in [18]. [19], [20] are proposed for data rate maximization. In [19] an algorithm to find the 3D locations of UAVs besides the user-BS associations and bandwidth allocations of the wireless backhaul to maximize the sum logarithmic rate of the users is proposed. Deployment algorithms for deploying a multi-relay network to maximize the end-to-end achievable rate are presented in [20]. An 
approach to find the optimum altitude of UAV that minimizes power loss, outage probability, and BER is presented in [21] while an approach to optimize the overall network delays is proposed in [22] All these works are proposed for UAV to assist the cellular network. However, the antenna down-tilting and low height of the cellular base station (BS) limits the ability of the UAV relay station to reach high altitudes due to the power constraint on the path between UAV and BS [23]. In other words, using the UAV as a relay station in the cellular system makes the UAV lose the advantage of deployment at optimum altitude which reflects directly on the coverage [7]. On the other hand, using UAV as an alternative to cellular BS is not a realistic solution because UAV has a drawback of the batteries' limited capacity which poses a challenge for the lifetime of its operation. Therefore, a UAV cannot be available for the entire duration of the operation as it is necessary to return for charging or replacing the battery. In this paper, Tethered UAV (TUAV) is proposed as an alternative to BS. TUAV is a UAV supplied by both power and data over a cable from a ground station (GS) as shown in Fig. 1. TUAV can achieve the main requirements of a reliable cellular BS in terms of endurance and backhaul link quality and the advantage of the UAV's high altitude as shown in Fig. 2. Recently, AT\&T's deployed the first TUAV to provide cellular coverage in Puerto Rico for the suffered regions after Hurricane Maria [24] which means that, TUAV can be a realistic alternative to the cellular BS. Therefore, we propose the TU2U2G system to replace the BS in the cellular system with TUAV to maximize the UAV relay station coverage. The contribution of this paper resides in several aspects:

- Propose a new relay system TU2U2G system. It uses the TUAV as a viable alternative to replace BS and provide seamless service over a cable that simultaneously supplies a stable power and a reliable wired data-link connection from a ground control station [6]. Compared to the BS, TUAV improves the system coverage due to its high attitude. Also, it overcomes the antenna down-tilting which increases the path loss between BS and UAV in the cellular System [3].

- Formulate the optimization problem to maximize UAV relay station coverage under the power budget and maximum UAV height constraints for the TU2U2G system. For simplicity, the 3D 
placement of the UAV is decoupled to the vertical and horizontal placement. Then, a 3D placement algorithm for the system is proposed.

- Numerical results are presented. TU2U2G the system shows better results than the cellular system in terms of optimum UAV height, maximum coverage radius, and maximum distance between BS and UAV.

The structure of the paper is organized as follows: Section 2 presents the system models. The 3D placement of UAV for maximum coverage is proposed in section 3. Numerical results are discussed in section 4. Finally, concluding remarks are in section 5.

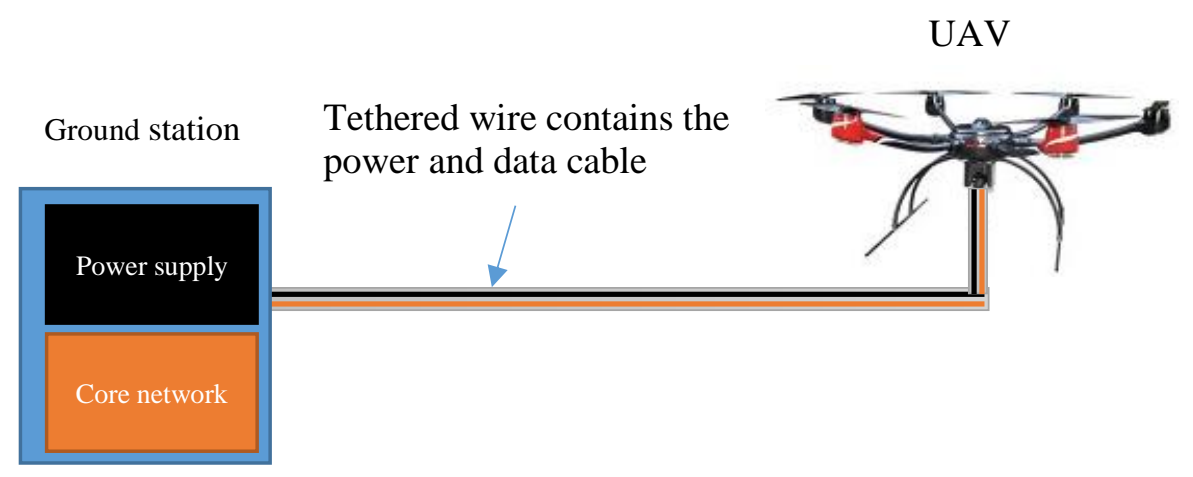

FIGURE 1. TUAV station components

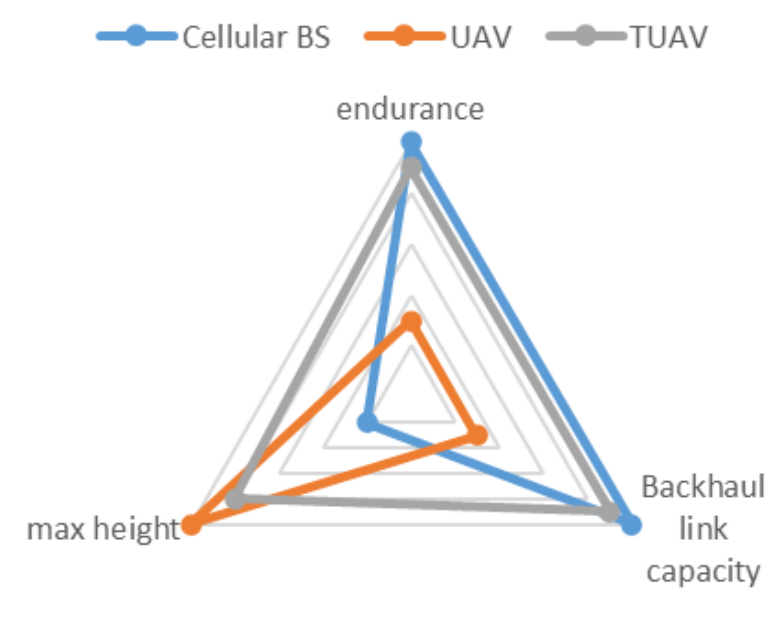

FIGURE 2. Cellular BS V.S. UAV V.S. TUAV 


\section{2 . System models}

The proposed TU2U2G system consists of TUAV, UAV, and MS. The ground distance between UAV and MS is R and $\theta$ is the elevation angle. The ground distance between ground TUAV and UAV is $d$ and $\alpha$ is the elevation angle. TU2U2G communication links are the A2G link and the TU2U link. The A2G link is between UAV and MS. TU2U link is between TUAV and UAV as shown in Fig.3. The UAV acts as an aerial base station or a relay to extend the wireless coverage. To formulate the optimization problem, it is essential to adopt the appropriate path loss models for the systems communication Links.

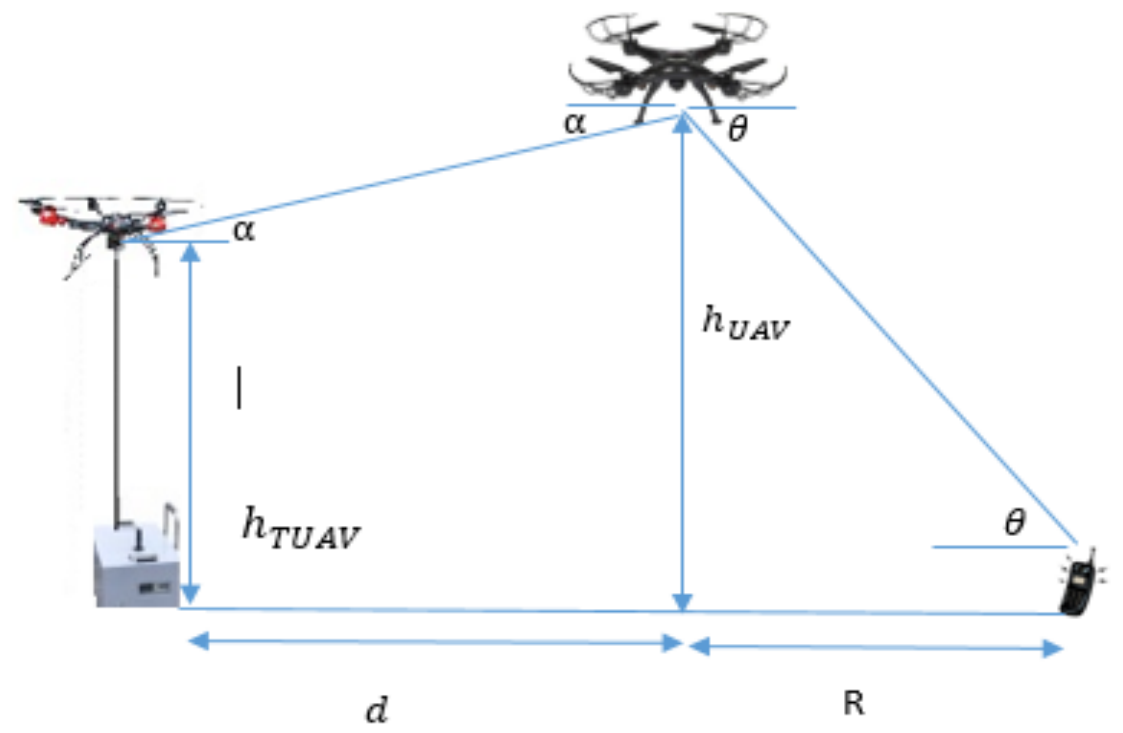

FIGURE 3.The TU2U2G system

\subsection{Channels models}

\subsubsection{A2G Channel}

The A2G path loss can be calculated by [4]:

$$
P L_{A 2 G}=F S P L+\eta_{\xi}
$$

FSPL represents the free space path loss between the UAV and a ground receiver. $\eta_{\xi}$ refers to LOS and NLOS excess path loss. The value of $\eta_{\xi}$ depends on the environment types [4]. The probability of having a LOS connection between the ground user and a UAV is given by [4]:

$$
\operatorname{PLOS}(\theta)=\frac{1}{1+a_{A 2 G} * e^{\left(-b_{A 2 G}\left[\theta-a_{A 2 G}\right]\right)}}
$$


Where $\mathrm{a}$ and $\mathrm{b}$ are constants that depend on the environment. Total path loss of the A2G link is presented in (4) by substituting with (2)\& (3) in(1).

$$
\begin{gathered}
\eta_{\xi}=\eta_{\mathrm{LOS}} P \operatorname{LOS}(\theta)+\eta_{\mathrm{NLOS}}(1-P \operatorname{LOS}(\theta)) \\
P L(R, \theta)=\frac{A}{1+a_{A 2 G} * e^{\left(-b_{A 2 G}\left[\theta-a_{A 2 G}\right]\right)}}+20 \log (R * \sec (\theta))+B
\end{gathered}
$$

\subsubsection{U2U Channel}

The TU2U path loss can be represented by a simple free-space propagation model [26], [27]. The free space path loss (FSPL) depends on the frequency and the distance between the transmitter and the receiver. The log-distance equation of FSPL is presented in (5).

$$
P L_{T U 2 U}=20 * \log (\mathrm{L})+20 * \log (\mathrm{f})-147.55
$$

Where $(\mathrm{L})$ is the distance in meters, and (f) is the frequency in $(\mathrm{Hz})$, and -147.55 is $-20 \log \left(\frac{4 \pi}{C}\right)$ where $\mathrm{C}$ is the speed of light. The distance (L) in (5) can be represented as a function of the ground distance between TUAV and UAV ( $\left.d_{T U 2 U}\right)$ and elevation angle as in (6).

$$
P L_{T U 2 U}\left(d_{T U 2 U}, \alpha\right)=-147.55+20 \log (\mathrm{f})+20 \log \left(d_{T U 2 U}\right)-20 \log \cos (\alpha)
$$

\subsection{UAV transmission power, SNR, and probability of coverage}

The transmit power $P_{T r}$ of UAV can be written as

$$
P_{T r}=P_{R x}+P L
$$

Where $P_{R x}$ is the received power (in $\left.\mathrm{dBm}\right) . P L$ is the path loss.

Therefore, the downlink SNR in $\mathrm{dB}$ is calculated as

$$
S N R=P_{T r}-P L-\sigma^{2}
$$

Where $\sigma^{2}$ is noise power. The coverage probability is the probability that a user can achieve SNR above the threshold $(T)$. Therefore, the user coverage probability can be defined as

$$
P_{c}=\mathrm{P}(S N R>T)
$$

Besides the SNR-based coverage approach, the coverage can be defined through path loss [7] [10]. 


$$
\begin{gathered}
S N R>T \\
P_{T r}-P L-\sigma^{2}>T \\
P L<P_{T r}-T_{A 2 G}-\sigma^{2} \\
P L<P L_{t h}
\end{gathered}
$$

Here, a user is considered to be covered by the UAV if $P L_{A 2 G}<P L_{t h(A 2 G)}$. Therefore, the UAV coverage radius can be mathematically defined as $\mathrm{R} \mid P L_{A 2 G}=P L_{t h(A 2 G)}$. It means that users at the distance $\mathrm{R}$ will have the same path loss $P L_{t h(A 2 G)}$, while users with a radius less than $R$ will experience path loss less than $P L_{t h(A 2 G)}$

- In the TU2U2G system, a UAV is considered to be inside the TUAV circular coverage if $P L_{T U 2 U}<$ $P L_{t h(T U 2 U)}$. Therefore, the TUAV coverage radius can be mathematically defined as $d_{T U 2 U} \mid P L_{T U 2 U}=$ $P L_{t h(T U 2 U)}$

\subsection{Problem formulation}

In this paper, the coverage is maximized by jointly optimizing the coverage radius of UAV $(R)$ and the distance between the TUAV and $\operatorname{UAV}\left(d_{T U 2 U}\right)$. In other words, our target is to maximize the number of covered users $(\mathrm{N})$ per UAV under the power budget and maximum UAV height constraints. The problem is formally written as follows:

$$
\max _{N, \beta_{i}, x_{i}, x_{U}, h_{U A V}, h_{T U A V}} N
$$

\section{Subject to constraints:}

$$
\begin{gathered}
\mathrm{C} 1: S N R_{A 2 G}>T \\
\mathrm{C} 2: S N R_{T u 2 U}>T \\
\mathrm{C} 3:\left(x_{i}-x_{U}\right)^{2}+\left(y_{i}-y_{U}\right)^{2} \leq R^{2}+M\left(1-\beta_{i}\right) \quad \forall i \in I \\
\mathrm{C} 4:\left(x_{U}-x_{T U}\right)^{2}+\left(y_{U}-y_{T U}\right)^{2} \leq d_{T U 2 U}^{2} \\
\mathrm{C} 5: P_{T r}(A 2 G) \leq P_{\max } \\
\mathrm{C} 6: P_{T r}(T U 2 U) \leq P_{\max }
\end{gathered}
$$




$$
\begin{gathered}
\text { C7: } h_{\text {UAVmin }} \leq h_{U A V} \leq h_{\text {UAVmax }} \\
\text { C8: } h_{\text {TUAVmin }} \leq h_{\text {TUAV }} \leq h_{\text {TUAVTax }}
\end{gathered}
$$

We aim to maximize the number of users $(\mathrm{N})$ covered by UAV in (14) under the constraints of transmit power and UAV height. C1 ensures that the user received SNR should be greater than the SNR threshold $T_{A 2 G}$. Therefore, the user is considered inside the UAV coverage. C2 imposes that UAV received SNR should be greater than the SNR threshold $T_{T U 2 U}$ for considering the UAV is inside the TUAV coverage. C3 assures that the user is inside the UAV coverage when located within the distance R from the UAV center $\left(x_{U}, y_{U}\right)$. Here $\beta \in\{0,1\}$ is a binary variable; $\mathrm{M}$ is a large constant to satisfy $\mathrm{C} 3$. $\mathrm{C} 4$ imposes that the UAV inside the TUAV coverage when located within the distance $d_{T U 2 U}$ from the TUAV center $\left(x_{T U}, y_{T U}\right)$. C5 assures that the user power should not be greater than the maximum allowable transmission power. C6 assures that the TUAV power should not be greater than the maximum allowable transmission power. C7 implies that the UAV altitude must be within the region $\left[h_{U A V \min } ; h_{\text {UAVmax }}\right] . \mathrm{C} 8$ implies that the UAV altitude must be within the region $\left[h_{\text {TUAVmin }} ; h_{\text {TUAVmax }}\right]$.

\section{3 . The proposed 3d placement of UAV}

It can be noticed that the UAV height is a joint variable between constrains equations of each optimization problem. Therefore, the 3D placement of the UAV is decoupled to the vertical and horizontal placement for simplicity. First, the vertical placement aims to find the optimum UAV heights for the maximum coverage radius. Since the coverage area of a UAV is considered as a circular disc. Therefore, the horizontal placement aims to find the center of the circular disc.

\subsection{Altitude Optimization for Maximum Coverage}

In the case of the TU2U2G system, the optimum height of the UAV is constrained by the maximum allowable path losses of the A2G link and TU2U link. Therefore, in the following the optimum UAV height for each communication link is separately obtained. Then, the final optimum UAV height for each system is obtained using the proposed algorithm. 


\subsubsection{Finding the UAV height for maximum coverage radius between UAV and ground user}

The optimal altitude that results in the maximum coverage region can be found by the first derivative $\frac{\partial R}{\partial \theta}=$ 0 . The optimal elevation angle $\theta_{O P T}$ depends on the type of environment [4]. The radius of the coverage area at $\theta_{O P T}$ can be calculated from (4). The $h_{U A V}$ that maximizes the coverage region is given by (24).

$$
\arg \max _{\theta} P L_{A 2 G}\left(h_{U A V}, \theta\right)=\left\{\theta_{O P T}: \frac{\pi \tan \left(\theta_{O P T}\right)}{9 \ln (10)}+\frac{a_{A 2 G} b_{A 2 G} A e^{\left(-b_{A 2 G}\left[\theta_{O P T}-a_{A 2 G}\right]\right)}}{\left[1+a_{A 2 G} e^{\left(-b_{A 2 G}\left[\theta_{O P T}-a_{A 2 G}\right]\right)}\right]^{2}}=0\right\}
$$

\section{Proof: Appendix A}

$$
h_{U A V}=R * \tan \left(\theta_{O P T}\right)+h_{m s}
$$

\subsubsection{Finding the UAV height for maximum distance between TUAV and UAV}

For a maximum allowable path loss between TUAV and UAV, the optimum angle $\alpha_{O P T}$ that maximizes $d_{T U 2 U}$ can be calculated from (25). The value of $\alpha_{O P T}=0$ which means that the maximum distance between TUAV and UAV is achieved when they are at the same level. The maximum distance of $d_{T U 2 U}$ at $\alpha_{O P T}$ can be calculated by (6). Then, optimum altitude difference between TUAV and UAV ( $\Delta \mathrm{h})$ is obtained by (26).

$$
\arg \max _{\alpha} P L_{T U 2 U}\left(h_{T U A V}, h_{U A V}, \alpha\right)=\left\{\alpha_{O P T}=0\right\}
$$

\section{Proof: Appendix B}

$$
\Delta h=d_{T U 2 U} * \tan \left(\alpha_{O P T}\right)
$$

\subsection{Horizontal placement of $U A V$}

The proposed horizontal placement aims to maximize the possible number of enclosed users considering the maximum allowable distance of $d_{T U 2 U}$. This is done by searching for the center of the coverage region under Constraints C3, C4. C3 assures that the user is inside the UAV coverage when located within the distance R from the UAV center. While $\mathrm{C} 4$ assures that the UAV is inside the TUAV coverage when located within the distance $d_{T U 2 U}$ from the TUAV center. 


\subsection{D placement algorithm}

In the proposed Algorithm, step (1) obtains the optimum $\mathrm{h}_{\mathrm{UAV}}$ for maximum coverage radius $\mathrm{R}$ under constraint $\mathrm{C} 1$. It is calculated from $\mathrm{A} 2 \mathrm{G}$ path loss by finding $\theta_{\mathrm{OPT}}$ which is obtained by solving the problem in (23). Then, substituting by $\theta_{\mathrm{OPT}}$ in (24) to calculate R. Then, substituting by $\theta_{\mathrm{OPT}}$ and $\mathrm{R}$ in (24) to calculate $\mathrm{h}_{\mathrm{UAV}}$.

Step (2) obtains the optimum $\Delta \mathrm{h}$ for maximum distance between UAV and TUAV under constraint C2. It is calculated by solving the problem in (25). Then, substituting by $\alpha_{\mathrm{OPT}}$ in (6) to calculate $d_{T U 2 U}$. Then, substituting by $\alpha_{\mathrm{OPT}}$ and $\mathrm{d}_{\mathrm{TU} 2 \mathrm{U}}(26)$ to calculate $\Delta \mathrm{h}$.

Step (3) calculates $h_{U A V}$ and $h_{T U A V}$ to satisfy the constraints $\mathrm{C} 7$ and C8. First, the maximum altitude difference between TUAV and UAV $\left(\Delta h_{\max }\right)$ is calculated by:

$$
\Delta h_{\max }=h_{U A V}-h_{T U A V_{\max }}
$$

Where $h_{T U A V_{\text {max }}}$ is the maximum altitude of TUAV. Then, the optimum of $h_{U A V}$ and $h_{T U A V}$ are found under the constraints of maximum and allowable height.

In Steps (4), (5), R and $d_{T U 2 U}$ are calculated for the new $h_{U A V}$ and $h_{T U A V}$ from (24) and (26) respectively.

Step (6) solves the problem (14) under constraints C3 and C4 to find the UAV horizontal placement. This is done by searching for the coverage region center $\left(x_{U}, y_{U}\right)$ that maximizes the number of users $(\mathrm{N})$. it is solved by branch and cut method [10].

Algorithm: Obtain Optimal 3D Location $\left(d, R, h_{T U A V}, h_{U A V}\right)$

Input: $A, B, a_{A 2 G}, b_{A 2 G}, \theta_{o p t}, h_{B S}, \eta_{L O S}, \eta_{N L O S}, h_{M S}, h_{T U A V_{\max }}, f, P_{U A V_{T x}}, P_{T U A V_{R x}}, P_{M S_{R x}}$

Output: $\left(d, R, h_{U A V}, h_{T U A V}\right)$

\section{Obtain $h_{U A V}$}

Finding $\theta_{O P T}$ by solving the problem in

(23).

Then, substituting by $\theta_{\mathrm{OPT}}$ in (24) to calculate R.

Then, substituting by $\theta_{\mathrm{OPT}}$ and $\mathrm{R}$ in (24) to calculate $h_{U A V}$.

\section{Obtain $\Delta h$}

Finding $\alpha_{O P T}$ by solving the problem in (25).

Then, substituting by $\alpha_{O P T}$ in (25) to calculate $d_{T U 2 U}$. 
Then, substituting by $\alpha_{O P T}$ and $d_{T U 2 U}(26)$ to calculate $\Delta h$.

3 Calculate $\Delta h_{\max }=$ absolute value of $\left(h_{U A V}-h_{T U A V_{\max }}\right)$

If $\left(\Delta h>\Delta h_{\max }\right)$ then

$$
\begin{aligned}
& \Delta h=\Delta h_{\max } \\
& h_{T U A V}=h_{T U A V_{\max }}
\end{aligned}
$$

else

$$
\Delta h=\Delta h
$$

If $\left(h_{U A V}>h_{B}\right)$ then

If $\left(\Delta h_{\max }=0\right)$ then

$$
h_{T U A V}=h_{U A V}
$$

else

$$
\begin{aligned}
& h_{U A V}=h_{T U A V_{\text {max }}}+\Delta h \\
& h_{T U A V}=h_{T U A V_{\text {max }}}
\end{aligned}
$$

end If

else

$$
h_{T U A V}=h_{U A V}+\Delta h
$$

end If

end If

4 Obtain $R$ from(24)

5 Obtain $d$ from(26)

6 Solve a problem: obtain UAV coverage center $\left(x_{U}, y_{U}\right)$ and users' coverage $N$ by solving the problem (14).

The worst-case complexity of the proposed algorithm is analyzed as follows. The complexity of Steps 1 to 5 is $O(n)$, Step 6 which is implements the branch and cut method is $O\left(2^{n}\right)$ and each branching node complexity is $O\left(n^{3.5} \log \left(\varepsilon^{-1}\right)\right)$ where $\varepsilon$ is the accepted duality gap. Therefore, the complexity of Algorithms is $O(n+$ $\left.2^{n} n^{3.5} \log \left(\varepsilon^{-1}\right)\right)$

\section{Numerical results}

In this section, numerical results of UAV placement for the TU2U2G system are presented. The optimum values of the distance between TUAV and UAV, and distance between BS and UAV, and the coverage Radius of the UAV are discussed. In addition to that, a comparison between the TU2U2G and cellular 
systems is presented. The system parameters are listed in Table1.

Table 1. The systems parameters

\begin{tabular}{cc}
\hline Frequency & $5.8 \mathrm{GHz}$ \\
\hline$h_{m S}$ & $2 \mathrm{~m}$ \\
$h_{B S}$ & $35 \mathrm{~m}$ \\
$h_{T U A V_{\text {max }}}$ & $100 \mathrm{~m}$ \\
$h_{B}$ & $60 \mathrm{~m}$ \\
$L_{r}$ & .6 \\
$G_{o}$ & 1.7 \\
$\gamma_{3}$ & 67 \\
\hline
\end{tabular}

To compare the TU2U2G system with the cellular system, both are simulated at different allowable path losses. Fig. 4 shows that, at the same allowable path loss, the distance between TUAV and UAV in the TU2U2G system is greater than the distance between BS and UAV in the cellular system. Also, the coverage radius of the UAV in the TU2U2G system is greater than the cellular system as shown in Fig. 5. It means that the TU2U2G system can extend the coverage better than the cellular system.

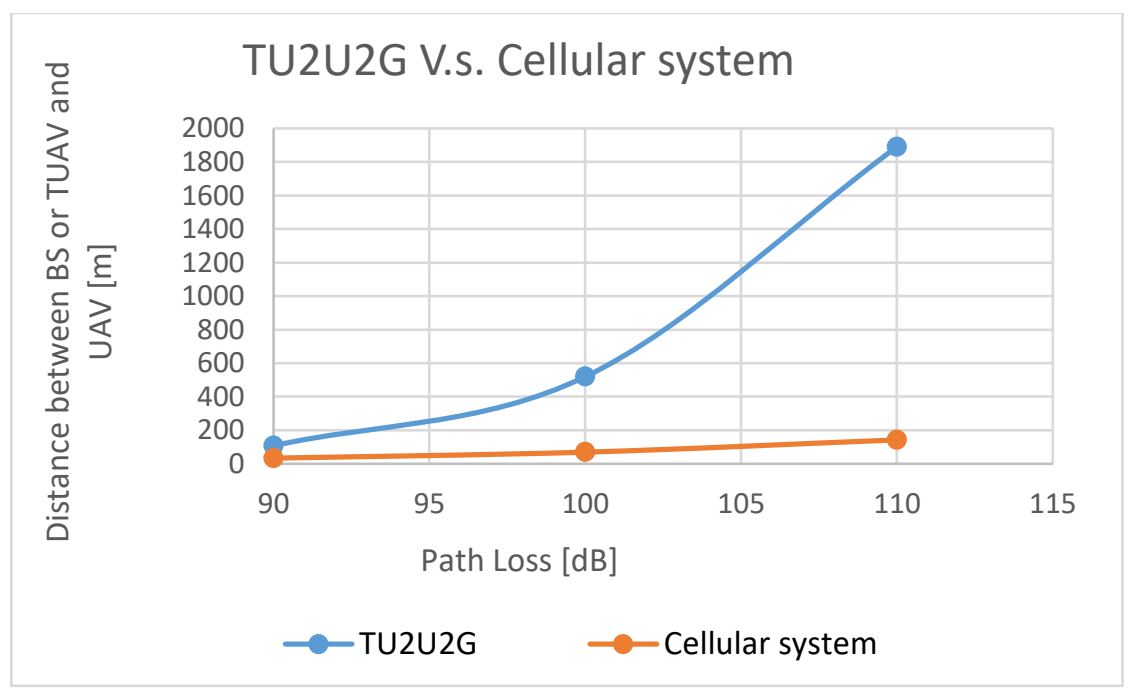

FIGURE 4. Distance between BS and UAV, and distance between TUAV and UAV at different path loss values 


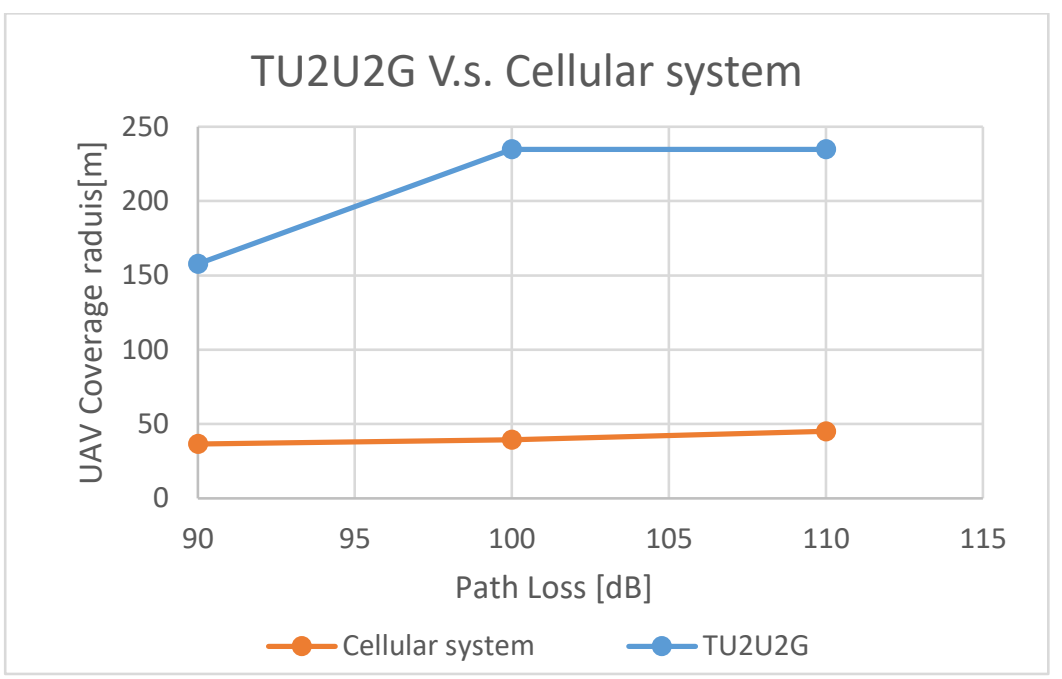

FIGURE 5. coverage radius of UAV for TU2U2G and Cellular system different path loss values

Fig. 6 and Fig. 7 show the 3D placement for the TU2U2G system and the cellular system respectively. The simulation is done at The $\mathrm{A} 2 \mathrm{G}$ path loss $=100 \mathrm{~dB}$. While A2A is set to different Path loss values $110 \mathrm{~dB}$, $115 \mathrm{~dB}, 120 \mathrm{~dB}$, and $125 \mathrm{~dB}$. The optimum altitude of the $\mathrm{A} 2 \mathrm{G}$ link for $\mathrm{PL}=100 \mathrm{~dB}$ is $225.9 \mathrm{~m}$ and the coverage radius is $208.7 \mathrm{~m}$. In the TU2U2G system, using TUAV with variable and high altitude allows the UAV to reach the optimum altitude of the A2G link. However, in the cellular system, the maximum coverage of the UAV is $70 \mathrm{~m}$. Fig. 8 shows the increase of UAV height and coverage with the increase of the TUAV height. On the other hand, the antenna down tilting and the low height of the Base station limit the UAV height to a max of $70 \mathrm{~m}$ which reduces the coverage radius of the UAV.

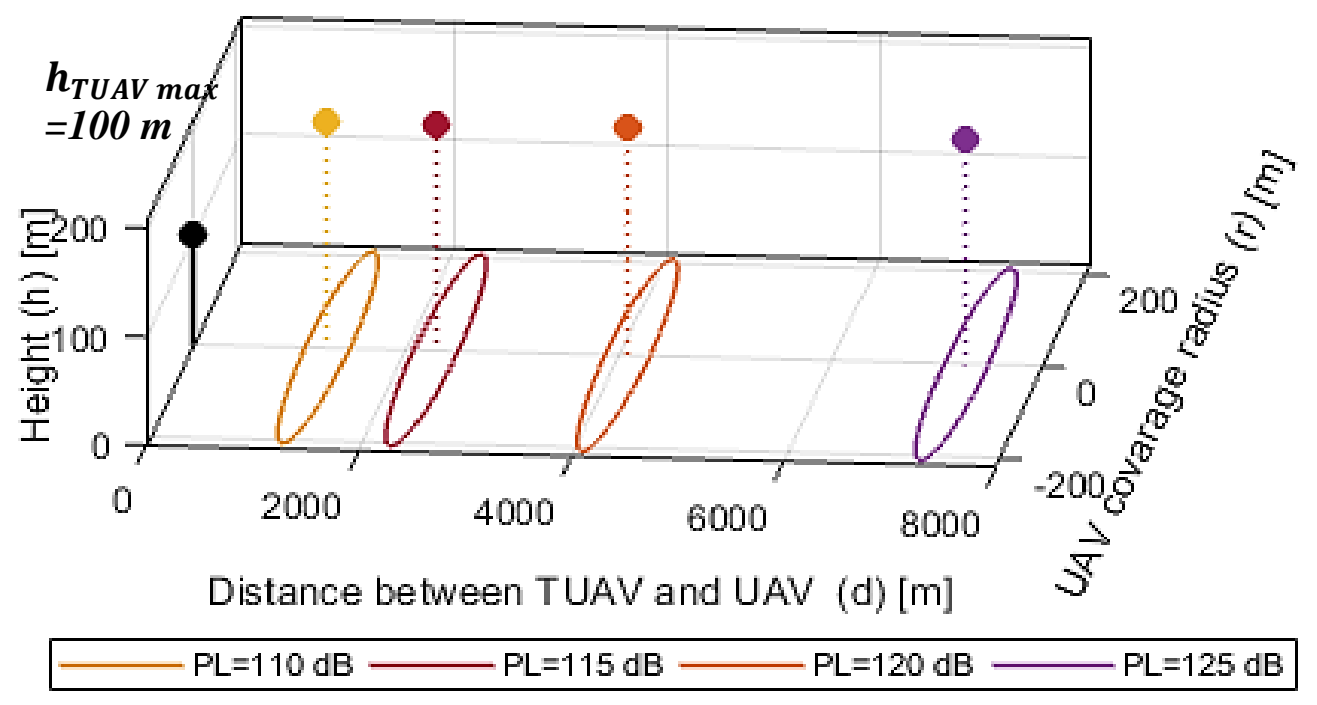

FIGURE 6. 3D placement of UAV for different Path losses 


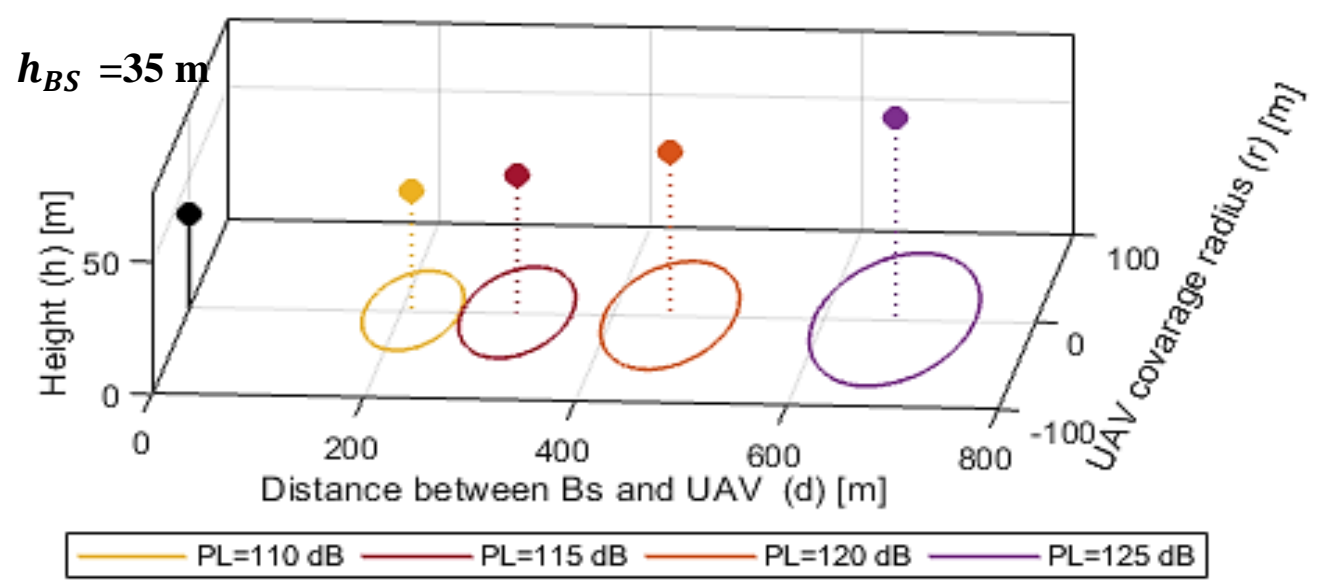

FIGURE 7. 3D placement of UAV for different Path losses

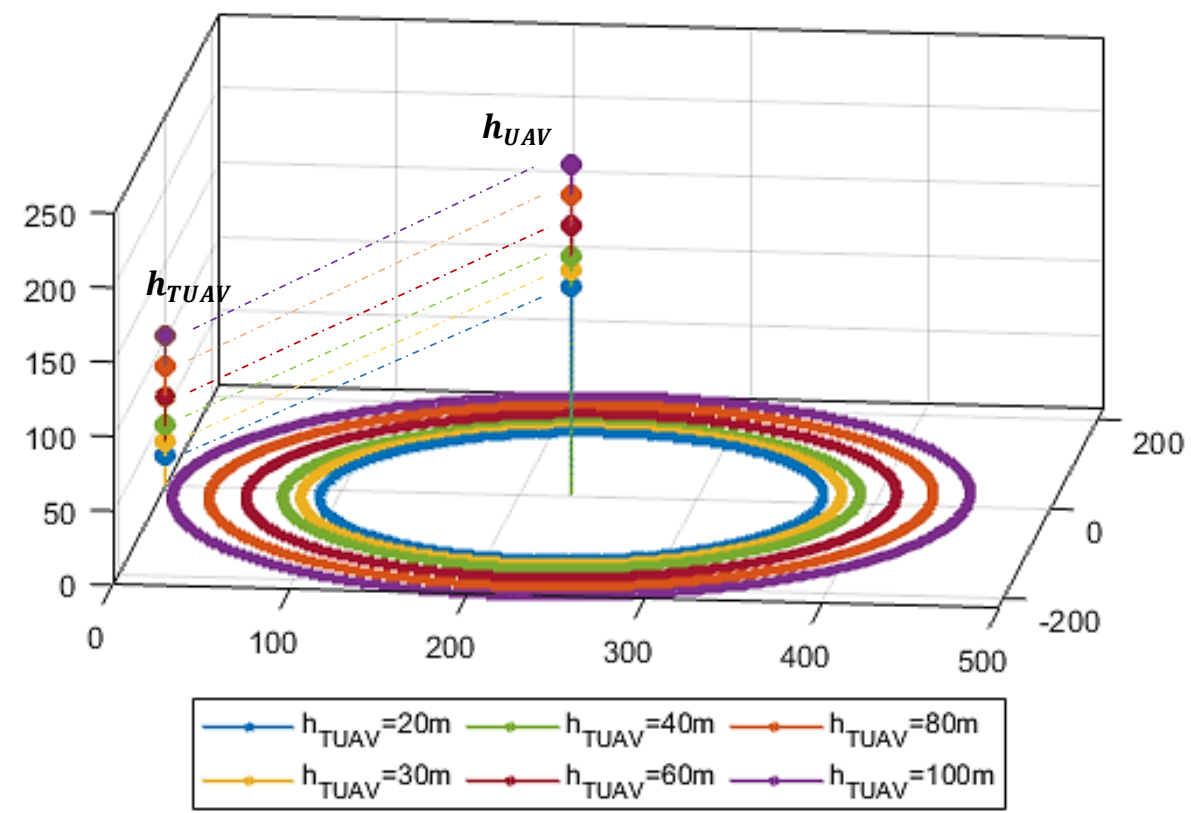

FIGURE 8. TUAV height Versus UAV height and coverage

\section{5 . Conclusion}

A new relay system TU2U2G that replaces BS with TUAV is proposed. In the proposed system, the TUAV has advantages of variable height up to $100 \mathrm{~m}$ and overcoming the problem of antenna down tilting. The 3D placement algorithm of UAV relay stations that jointly optimize the transmitting power and relaying distance for the coverage maximization is proposed. TU2U2G the system shows better results than the cellular system in terms of optimum UAV height, maximum coverage radius, and maximum distance between BS and UAV. 


\section{APPENDIX}

\section{Appendix A:}

- The optimum height of $\operatorname{UAV}(h)$ for maximum coverage radius $(R)$ can be found by differentiating $R$ with respect to $h \quad\left(\frac{\partial R}{\partial h}\right)=\frac{\partial R}{\partial \theta} * \frac{\partial \theta}{\partial h}=0$. Since $\frac{\partial \theta}{\partial h}=\frac{\partial \tan ^{-1}\left(\frac{h}{R}\right)}{\partial h}=\frac{R}{R^{2}+h^{2}}>1$, then optimum altitude can be achieved by searching for the optimum elevation angle $\alpha_{O P T}$ that maximizes $(R)$. It is calculated by the first derivative

$$
\begin{gathered}
\frac{\partial R}{\partial \theta}=0 \\
\frac{\pi \tan \left(\theta_{O P T}\right)}{9 \ln (10)}+\frac{a_{A 2 G} b_{A 2 G} A e^{\left(-b_{A 2 G}\left[\theta_{O P T}-a_{A 2 G}\right]\right)}}{\left[1+a_{A 2 G} e^{\left(-b_{A 2 G}\left[\theta_{O P T}-a_{A 2 G}\right]\right)}\right]^{2}}=0
\end{gathered}
$$

\section{Appendix B:}

- The maximum distance between TUAV and UAV $\left(d_{T U 2 U}\right)$ can be found by differentiating $d_{T U 2 U}$ with respect to $\Delta h \quad\left(\frac{\partial d_{T U 2 U}}{\partial(\Delta h)}\right)=\frac{\partial d_{T U 2 U}}{\partial \alpha} * \frac{\partial \alpha}{\partial(\Delta h)}=0$. Since $\frac{\partial \alpha}{\partial(\Delta h)}=\frac{\partial \tan ^{-1}\left(\frac{\Delta h}{d}\right)}{\partial(\Delta h)}=\frac{d_{T U 2 U}}{d_{T U 2 U^{2}}+\Delta h^{2}}>1$, then optimum altitude can be achieved by searching for the optimum elevation angle $\alpha_{O P T}$ that maximizes $\left(d_{T U 2 U}\right)$. It is calculated by the first derivative (6) with respect to $\alpha$

$$
\begin{gathered}
\frac{\partial d_{T U 2 U}}{\partial \alpha}=0 \\
\frac{\partial d_{T U 2 U}}{\partial \alpha}=\frac{\pi \tan \left(\alpha_{O P T}\right)}{9 \ln (10)}=0
\end{gathered}
$$

\section{References}

[1] Mozaffari, M., Saad, W., Bennis, M., et al., "A tutorial on UAVs for wireless networks: applications, challenges, and open problems," IEEE Communications Surveys \& Tutorials, vol. 21, no. 3, pp. 23342360, 2019.

[2] Gupta, Lav and Jain, Raj and Vaszkun, Gabor, "Survey of important issues in UAV communication networks," IEEE Communications Surveys \& Tutorials, vol. 18, no. 2, pp. 1123--1152, 2015.

[3] Al-Hourani, Akram and Gomez, Karina, "Modeling cellular-to-UAV path-loss for suburban environments," IEEE Wireless Communications Letters, vol. 7, no. 1, pp. 82-85, 2017.

[4] Al-Hourani, A., Kandeepan, S., Lardner, S., "Optimal LAP altitude for maximum coverage," IEEE Wireless Communications Letters, vol. 3, no. 6, pp. 569-572, 2014.

[5] AT\&T, "AT\&T’s First Official Deployment of Cell On Wings In Puerto Rico," 2017. [Online]. Available: https://t2m.io/xVDCQmjd. [Accessed 3011 2021]. 
[6] Bushnaq, Osama M and Kishk, Mustafa A and Celik, Abdulkadir and Alouini, Mohamed-Slim and AlNaffouri, Tareq Y, "Optimal deployment of tethered drones for maximum cellular coverage in user clusters," IEEE Transactions on Wireless Communications, vol. 20, no. 3, pp. 2092-2108, 2020.

[7] Shakoor, Shanza and Kaleem, Zeeshan and Do, Dinh-Thuan and Dobre, Octavia A and Jamalipour, Abbas, "Joint optimization of UAV 3D placement and path loss factor for energy efficient maximal coverage," IEEE Internet of Things Journal, 2020.

[8] Cui, Jian and Shakhatreh, Hazim and Hu, Bo and Chen, Shanzhi and Wang, Chuanan, "Power-efficient deployment of a UAV for emergency indoor wireless coverage," IEEE Access, vol. 6, pp. 73200-73209, 2018.

[9] Alzenad, Mohamed and El-Keyi, Amr and Lagum, Faraj and Yanikomeroglu, Halim, "3-D placement of an unmanned aerial vehicle base station (UAV-BS) for energy-efficient maximal coverage," IEEE Wireless Communications Letters, vol. 6, no. 4, pp. 434-437, 2017.

[10] Alzenad, Mohamed and El-Keyi, Amr and Yanikomeroglu, Halim, "3-D placement of an unmanned aerial vehicle base station for maximum coverage of users with different QoS requirements," IEEE Wireless Communications Letters, vol. 7, no. 1, pp. 38-41, 2017.

[11] Sun, Yan and Xu, Dongfang and Ng, Derrick Wing Kwan and Dai, Linglong and Schober, Robert, "Optimal 3D-trajectory design and resource allocation for solar-powered UAV communication systems," \{IEEE Transactions on Communications, vol. 67, no. 6, pp. 4281-4298, 2019.

[12] Wang, Haichao and Ren, Guochun and Chen, Jin and Ding, Guoru and Yang, Yijun, "Unmanned aerial vehicle-aided communications: Joint transmit power and trajectory optimization," IEEE Wireless Communications Letters, vol. 7, no. 4, pp. 522-525, 2018.

[13] Wu, Qingqing and Zeng, Yong and Zhang, Rui, "Joint trajectory and communication design for multiUAV enabled wireless networks," IEEE Transactions on Wireless Communications, vol. 17, no. 3, pp. 2109-2121, 2018.

[14] Cai, Yuanxin and Wei, Zhiqiang and Li, Ruide and Ng, Derrick Wing Kwan and Yuan, Jinhong, "Joint trajectory and resource allocation design for energy-efficient secure UAV communication systems," IEEE Transactions on Communications, vol. 68, no. 7, pp. 4536-4553, 2020.

[15] El Hammouti, Hajar and Benjillali, Mustapha and Shihada, Basem and Alouini, Mohamed-Slim, "Learnas-you-fly: A distributed algorithm for joint 3D placement and user association in multi-UAVs networks," IEEE Transactions on Wireless Communications, vol. 18, no. 12, pp. 5831-5844, 2019.

[16] Huang, Min and Huang, Lei and Zhong, Shida and Zhang, Peichang, "UAV-Mounted Mobile Base Station Placement via Sparse Recovery," IEEE Access, vol. 8, pp. 71775-71781, 2020.

[17] Zhang, Jun and Liang, Fengzhu and Li, Bin and Yang, Zheng and Wu, Yi and Zhu, Hongbo, "Placement optimization of caching UAV-assisted mobile relay maritime communication," China Communications, vol. 17, no. 8, pp. 209-219, 2020.

[18] Li, Yue and Feng, Guangsheng and Ghasemiahmadi, Mohammad and Cai, Li, "Power allocation and 3-D placement for floating relay supporting indoor communications," IEEE Transactions on Mobile Computing, vol. 18, no. 3, pp. 618-631, 2018.

[19] Chen, Junting and Gesbert, David, "Optimal positioning of flying relays for wireless networks: A LOS map approach," in 2017 IEEE international conference on communications (ICC), 2017.

[20] Larsen, Erlend and Landmark, Lars and Kure, "Optimal UAV relay positions in multi-rate networks," in 2017 Wireless Days, 2017.

[21] Y. a. Z. R. a. L. T. J. Zeng, "Throughput maximization for UAV-enabled mobile relaying systems," IEEE Transactions on Communications, vol. 64, no. 12, pp. 4983-4996, 2016.

[22] Kalantari, Elham and Bor-Yaliniz, Irem and Yongacoglu, Abbas and Yanikomeroglu, Halim, "User association and bandwidth allocation for terrestrial and aerial base stations with backhaul considerations," in IEEE, 2017. 
[23] Chattopadhyay, Arpan and Ghosh, Avishek and Kumar, Anurag, "Asynchronous stochastic approximation based learning algorithms for as-you-go deployment of wireless relay networks along a line," IEEE Transactions on Mobile Computing, vol. 17, no. 5, pp. 1004-1018, 2017.

[24] Chen, Yunfei and Feng, Wei and Zheng, Gan, "Optimum placement of UAV as relays," IEEE Communications Letters, vol. 22, no. 2, pp. 248-251, 2017.

[25] Sharma, Vishal and Sabatini, Roberto and Ramasamy, Subramanian, "UAVs assisted delay optimization in heterogeneous wireless networks," IEEE Communications Letters, vol. 20, no. 12, pp. 2526-2529, 2016.

[26] Ahmed, Nadeem and Kanhere, Salil S and Jha, Sanjay, "On the importance of link characterization for aerial wireless sensor networks," IEEE Communications Magazine, vol. 54, no. 5, pp. 52-57, 2016.

[27] Allred, Jude and Hasan, Ahmad Bilal and Panichsakul, Saroch and Pisano, William and Gray, Peter and Huang, Jyh and Han, Richard and Lawrence, Dale and Mohseni, Kamran, "Sensorflock: an airborne wireless sensor network of micro-air vehicles," in Proceedings of the 5th international conference on Embedded networked sensor systems, 2007. 